\title{
La pensée enseignante dans une expérience de tutorat en ligne
}

Vers un agir tutoral à distance

Catherine Muller

\section{(2) OpenEdition Journals}

Édition électronique

URL : http://journals.openedition.org/rdlc/758

DOI : $10.4000 /$ rdlc.758

ISSN : 1958-5772

Éditeur

ACEDLE

Référence électronique

Catherine Muller, "La pensée enseignante dans une expérience de tutorat en ligne », Recherches en didactique des langues et des cultures [En ligne], 12-2 | 2015, mis en ligne le 20 novembre 2015, consulté le 19 avril 2019. URL : http://journals.openedition.org/rdlc/758 ; DOI : 10.4000/rdlc.758

Ce document a été généré automatiquement le 19 avril 2019

\section{(9) $\odot \Theta \Theta$}

Recherches en didactique des langues et des cultures is licensed under a Creative Commons AttributionNonCommercial-NoDerivatives 4.0 International License 


\title{
La pensée enseignante dans une expérience de tutorat en ligne
}

\author{
Vers un agir tutoral à distance
}

\author{
Catherine Muller
}

\section{Introduction}

1 Comme le souligne Cicurel, "Un enseignant entre dans une classe avec un passé, une expérience, des doctrines personnelles sur l'enseignement" (2011: 16). Qu'en est-il lorsque l'action enseignante ne se déroule pas en présentiel mais à distance? Comment les convictions personnelles engrangées dans l'expérience passée d'apprenant en présentiel, voire d'enseignant en présentiel, influencent-elles l'agir enseignant à distance? Nous proposons de réfléchir à la fabrique de l'action enseignante dans un dispositif à distance impliquant des outils numériques. Notre étude prendra appui sur un projet de télécollaboration asynchrone au cours duquel des étudiants inscrits en master 2 professionnel "didactique du FLE ${ }^{1}$ et interculturalité" à l'université Paris Descartes ont exercé le rôle de tuteurs en ligne pour des étudiants hongkongais. La visée ici est de comprendre la construction progressive d'un agir tutoral à distance. Deux paramètres sont à prendre en compte : le dispositif se déroule en ligne et les tuteurs sont novices pour la plupart. Que signifie pour eux le fait d'être tuteurs en ligne? Comment construisent-ils une analogie entre ce dispositif nouveau pour eux et leurs précédentes expériences? Est-il possible pour les tuteurs de s'appuyer sur des routines pour agir? Si ce n'est pas le cas, dans quelles ressources puisent-ils leur agir tutoral? Comment se fondent-ils sur la formation qu'ils ont reçue ? Comment émergent de nouvelles pratiques pour les tuteurs en ligne et à quelles réflexions donnent-elles lieu? Pour tenter de répondre à ces questions, nous étudierons des carnets de bord rédigés par les apprentis tuteurs. Au cours de ces écrits réflexifs, les tuteurs commentent leur action en ligne. Après avoir présenté la notion d'agir enseignant, nous exposerons le contexte de l'étude. L'analyse des données s'effectuera en trois temps. Nous montrerons d'abord comment se 
construit une action conjointe, avant de mettre en exergue l'importance de l'analogie, qui apparait comme un fondement de l'action enseignante. Notre étude abordera pour terminer l'émergence de routines liées à de nouvelles pratiques chez les apprentis tuteurs.

\section{La construction de l'agir enseignant}

Notre étude s'inscrit dans la continuité des recherches sur l'agir professoral qui s'attachent à prendre en compte le point de vue des enseignants. Cela passe notamment par un intérêt pour les modèles implicites des formateurs. Nous nous efforcerons de mettre en relation cette approche avec la question posée ici, celle de la construction d'un agir tutoral à distance.

\section{Les recherches sur l'agir enseignant : une prise en compte du point de vue de l'enseignant}

Cicurel désigne par agir professoral "l'ensemble des actions verbales et non verbales, préconçues ou non, que met en place un professeur pour transmettre et communiquer des savoirs ou un "pouvoir-savoir" à un public donné dans un contexte donné" (2011: 48). Il convient de souligner l'importance de l'intentionnalité dans la pratique enseignante (Cicurel, 2005 ; Cicurel, 2007a). L'action est planifiée mais elle se construit également localement (van Lier, 1996: 215). Le professeur est constamment amené à prendre des décisions dans l'urgence, ce qui implique un engagement intense (van Lier, 1996 : 31). Les obstacles qui se présentent peuvent conduire à une réorientation du déroulement prévu.

Une telle approche centrée sur l'enseignant a été développée dans le monde anglo-saxon sous l'expression de "teacher cognition" (Woods, 1996; Borg, 2003, 2009). Ce courant de recherche s'attache au point de vue des enseignants, c'est-à-dire leur propre "perception et interprétation de ce qui se passe en classe"2 (Woods, 1996: 22). Il s'agit ainsi d'une "étude ethnographique des enseignants"s (Woods, 1996: 47), qui vise à comprendre ce qu'ils " pensent, savent et croient"4 (Borg, 2009). Cette perspective est dite "émique" : ce n'est pas le point de vue de l'observateur extérieur qui est pris en compte, mais bien celui du participant en contexte.

\section{L'importance des modèles implicites}

Dans ce cadre, il convient de se demander sur quoi se fonde l'agir professoral. La notion de répertoire didactique désigne "le stock de savoirs, d'expériences antérieures, de modèles intériorisés d'un enseignant" (Cicurel, 2011: 218-219). Les recherches sur la pensée enseignante portent un intérêt particulier aux croyances, aux théories implicites, aux convictions et aux savoirs des enseignants (Woods, 1996 ; Cambra Giné, 2003).

Les enseignants disposent de modèles implicites (Tochon, 1993: 68) et c'est à la lumière de leurs croyances à propos de l'enseignement/apprentissage qu'ils interprètent une situation de classe (Woods, 1996: 69). Comme les apprenants, les enseignants ont leur propre idée de ce à quoi doit ressembler un cours réussi et de la façon dont les langues devraient être apprises et enseignées (van Lier, ([1988] 1990: 179). Ces "théories 
personnelles" (Cicurel, 2011: 115) des professeurs se forgent au fur et à mesure de leur formation et de leur expérience professionnelle mais aussi personnelle.

\section{L'agir tutoral à distance}

7 Les recherches sur l'agir professoral portent le plus souvent sur des dispositifs en présentiel, dans des classes. Nous allons nous intéresser ici au cas du tutorat en ligne, fondé sur une relation d'aide avec un conseiller qui accompagne le travail et propose une remédiation (Denis, 2003; Bourdet, 2006; Rodet, 2011; Bertin, 2013). Dans de tels dispositifs, d'autres ressources matérielles sont à l'œuvre. On se situe dans un cas de " communication pédagogique médiatisée" (Dejean-Thircuir \& Mangenot, 2006) ou d'interactions pédagogiques en ligne (Mangenot, 2011). Les interactions sont bien présentes, mais elles se produisent à distance, que ce soit sous une forme écrite ou orale, en mode synchrone ou asynchrone, comme l'indique Nissen (2011: 164).

Dans la télécollaboration que nous allons étudier, les échanges se sont uniquement déroulés en mode asynchrone. Un dispositif de ce type pose un certain nombre de questions concernant l'action enseignante : Qu'est-ce que l'agir tutoral lorsque l'action du tuteur ne se manifeste pas par une co-présence physique avec les apprenants? À quoi correspond l'action d'un tuteur à distance s'il ne fait pas cours en présence ? Comment se construit l'action tutorale à distance lorsque les tuteurs ne peuvent ni voir leurs apprenants ni communiquer en direct avec eux?

\section{Contexte de l'étude et démarche méthodologique}

9 Après avoir présenté les caractéristiques du dispositif de télécollaboration dont il sera question ici, nous exposerons le corpus qui sera étudié, ainsi que les questions de recherche retenues.

\section{Présentation du dispositif de télécollaboration}

Les projets de télécollaboration permettent à des classes distantes d'échanger en ligne. De tels dispositifs peuvent prendre différentes formes. Celui que nous avons mis en place s'inspire directement de la recherche-action Le français en (première) ligne, initiée par Develotte et Mangenot (voir par exemple Develotte, Mangenot \& Zourou, 2007), dans lequel des étudiants en didactique du FLE exercent le rôle de tuteurs en ligne pour des apprenants. Le partenariat ${ }^{5}$ dont il sera question ici a réuni pendant deux mois 25 tuteurs étudiants en master 2 professionnel "didactique du FLE et interculturalité" à l'université Paris Descartes et 13 apprenants de l'université baptiste de Hong Kong, inscrits en licence 2 d'études européennes.

11 Pour les apprentis tuteurs qui assurent la conception et le suivi de tâches, ce dispositif apporte une initiation au tutorat en ligne et un perfectionnement du maniement des outils numériques pour l'enseignement/apprentissage du FLE. Pour les apprenants de FLE, l'expérience offre un suivi personnalisé et apporte une possibilité d'échanges authentiques avec des étudiants natifs ou experts de la langue française. Le nombre de participants de part et d'autre a conduit à des proportions inhabituelles de deux tuteurs pour un apprenant. 

utilisé pour communiquer de façon asynchrone. Chaque trio pédagogique (deux tuteurs pour un apprenant) a bénéficié de son espace propre, sur lequel les tuteurs indiquent les tâches, les apprenants y répondent et les tuteurs apportent du feedback via un forum et des fichiers joints. La télécollaboration s'inscrit dans une pédagogie de projet visant à l'élaboration d'un blog dédié à la vie étudiante en France et à Hong Kong. Apprenants comme tuteurs doivent publier des témoignages sur une série de thèmes (logement, voyages, sorties, etc. $)^{6}$.

\section{Le recours à des écrits réflexifs}

13 Dans la mesure où les échanges se sont uniquement produits en mode asynchrone, il n'a pas été possible de mettre en place un dispositif d'auto-confrontation à la manière de Guichon (2011) afin de confronter des tuteurs en ligne aux enregistrements de leurs échanges avec les apprenants. Pour disposer de leur regard réflexif, nous avons demandé aux tuteurs de rédiger un carnet de bord au fur et à mesure des 8 semaines du projet ou un bilan réflexif final. Ce texte, à remettre à la fin du projet, a fait l'objet d'une évaluation notée. Dans le cadre de cette réflexion personnelle, les étudiants ont été invités à présenter leur propre interprétation des échanges ${ }^{7}$, en insistant sur ce qui leur a convenu, ce qui leur a manqué, sur les aspects positifs, les limites, les obstacles rencontrés et les solutions trouvées. Il s'agissait pour eux de s'interroger sur les apports $\mathrm{du}$ projet dans le cadre de leur formation. Leur regard sur le dispositif, leurs représentations sur l'enseignement à distance, les Tice et le tutorat ont-ils évolué ? Quelles spécificités ont-ils perçues dans le dispositif (rôle du tuteur, élaboration et suivi des activités, relation tuteur/apprenant), par rapport à leur expérience d'apprenant et/ ou d'enseignant en présentiel ? Le texte pouvait également porter sur les modalités des échanges, sur l'usage de la plateforme, le tutorat à deux pour un apprenant et l'utilisation des Carnets 2 Paris Descartes.

Il ne s'agit pas d'un discours interactif ou d'une co-construction comme lors d'un entretien. Les étudiants commentent a posteriori leur agir tutoral en ligne. La tenue d'un journal de bord est préconisée par Bailey (1990), Tochon (1993: 198) ou encore Guichon (2012 : 201-202). Il convient de prendre en compte le contexte spécifique de rédaction de ces textes (évaluation dans le cadre d'un cours), mais l'on peut avancer que les carnets de bord et les bilans réflexifs constituent une façon d'avoir accès au "monde intérieur" des tuteurs.

Nous proposons ici une étude de cas focalisée sur les carnets de bord d'un binôme de tuteurs en particulier. Il s'agit de Yann", âgé de 24 ans, qui a une expérience de deux stages de FLE avec des prises en main, et de Sabrina, âgée de 22 ans, qui dispose d'une expérience de tutorat en présentiel pour apprenants allophones et d'une expérience d'enseignement du FLE pendant 3 mois. Ils sont en charge du tutorat d'un apprenant hongkongais, Larry. Ces tuteurs ont été sélectionnés d'une part parce qu'ils sont encore novices dans l'enseignement du FLE ${ }^{9}$ et d'autre part parce qu'ils ont tous les deux rédigé un carnet de bord et non un bilan réflexif, ce qui permettra d'étudier l'évolution de leur réflexion.

Recherches en didactique des langues et des cultures, 12-2 | 2015 


\section{Questions de recherche}

16 Nous posons que les carnets de bord sont un moyen d'accéder à la construction en direct de l'agir tutoral à distance, qui différerait de l'agir enseignant et serait inédit pour eux. Nous nous attacherons ici aux réflexions, aux démarches et aux pratiques acquises par ces deux tuteurs novices dans le cadre du projet de télécollaboration. L'étude des carnets de bord visera à comprendre la façon dont les tuteurs se représentent leur action. Nos questions de recherche sont les suivantes : Comment les carnets de bord révèlent-ils la construction progressive d'un agir tutoral à distance? En d'autres termes, comment se fabrique l'action de tuteurs en ligne? Dans quelles ressources les tuteurs puisent-ils leur agir tutoral ? Pour répondre à ces questions, nous procéderons à une analyse qualitative des carnets de bord et chercherons à prendre en compte le développement longitudinal en prêtant une attention particulière à la semaine de rédaction des réflexions. Nous opterons pour une approche compréhensive et privilégierons une perspective émique.

\section{La construction d'une action conjointe}

La première partie de notre analyse porte sur la construction d'une "action conjointe" (Sensevy \& Mercier (dir.), 2007) avec l'apprenant, mais également avec l'autre tuteur du groupe.

\section{Une action qui s'adapte à l'apprenant}

Il convient de rappeler que cette situation de tutorat est assez exceptionnelle, dans la mesure où deux tuteurs se chargent de la conception et du suivi des tâches pour un seul apprenant. Cela entraîne un fort accent sur l'adaptation à l'apprenant et la personnalisation des tâches.

\section{Analyse des besoins et progression}

Les tuteurs cherchent à cerner les besoins de leur apprenant pour construire une progression. Pour cela, ils évaluent son niveau à travers sa réalisation des tâches. À la semaine 2, Sabrina manifeste son étonnement lié aux réponses correctes de Larry pour l'activité de compréhension :

\section{Carnet de bord Sabrina, semaine 2 :}

Il a répondu correctement à presque toutes les questions de compréhension ; je suis surprise de son niveau linguistique d'autant plus qu'il a commencé à apprendre le français il y a seulement un an. Je me suis même demandé, avant d'arriver aux réponses fausses qu'il a données, si le travail que je lui avais proposé n'était pas trop facile. C'est assez difficile de pouvoir situer correctement le niveau de l'apprenant que l'on aide.

Cet extrait, produit au début du dispositif, manifeste le décalage entre les attentes de Sabrina et les performances de l'apprenant. La tutrice avait supposé un niveau moins avancé en se fondant sur la durée d'apprentissage du français: ses estimations peuvent être liées à sa formation en didactique du FLE, ainsi qu'à ses pratiques enseignantes ou apprenantes. La présence d'erreurs dans le travail rendu par l'apprenant ("presque toutes 
les questions", "réponses fausses qu'il a données") apparaît comme un soulagement pour la tutrice. Celle-ci s'interroge en effet sur le caractère approprié de la tâche, ce qui témoigne de ses représentations, que l'on pourrait reformuler ainsi : une tâche appropriée donne lieu à des réponses incorrectes, sinon c'est "trop facile". On le voit, la question de la facilité ou de la difficulté des tâches demandées est une source de préoccupation pour la tutrice. Mais cette question de la difficulté apparaît également dans la caractérisation du rôle de tuteur: "C'est assez difficile".

21 C'est ainsi qu'à la semaine suivante, elle "rectifie le tir", en augmentant la complexité :

\title{
Carnet de bord Sabrina, semaine 3 :
}

Mes exercices sont un peu plus complexes que la première fois et jouent beaucoup sur les implicites, ainsi que sur les antithèses présentes dans le texte travaillé.

Sabrina souligne les différents éléments qui peuvent être une source de difficulté pour l'apprenant. Elle se fonde donc entièrement sur les productions de Larry pour construire et faire évoluer ses tâches. De la même manière, à la semaine 5 , Sabrina prend appui sur les difficultés de son apprenant concernant le lexique pour proposer une tâche :

\section{Carnet de bord Sabrina, semaine 5 :}

\begin{abstract}
J'ai remarqué depuis la dernière fois où je m'étais chargé de l'activité de compréhension, que Larry avait quelques difficultés à percevoir certaines subtilités du lexique contenu dans les articles de presse proposés. J'étais tout de même contente, malgré les quelques erreurs qu'il a fait, de pouvoir cibler une activité qui lui pose quand même un peu problème car, d'abord, c'est une activité qui lui profitera à l'avenir, mais aussi parce que j'ai pu enfin cibler un de ses besoins.
\end{abstract}

La tutrice cherche plus particulièrement les points qui posent problème à l'apprenant. Le verbe "cibler", précédé du verbe de modalité "pouvoir", est employé à deux reprises : il témoigne de l'importance que la tutrice accorde à l'adaptation précise aux besoins de son apprenant. Sabrina exprime une satisfaction, qu'elle estime paradoxale ("tout de même"), liée aux difficultés rencontrées par Larry. On l'a vu, les erreurs témoignent selon elle de la pertinence de l'activité.

Les tuteurs commentent ainsi les productions de leur apprenant et évaluent sa progression :

Carnet de bord Yann, semaine 7 :

Cela ne semble pas avoir perturbé l'apprenant puisqu'il n'a pas eu de problème pour lire la vidéo ou comprendre l'audio: ces réponses étaient pour la plupart juste. Encore une fois, son témoignage était vraiment intéressant. Je sens qu'il a progressé par rapport à ses premiers témoignages, il s'engage plus dans son discours, il explique et détaille vraiment bien, presque à la française.

Dans cet extrait rédigé vers la fin du dispositif, Yann souligne la justesse des réponses et l'intérêt des productions de Larry. Les critères qu'il retient ("il s'engage plus dans son discours, il explique et détaille vraiment bien, presque à la française") semblent influencés à la fois par sa formation en didactique du FLE mais aussi par son expérience d'apprenant de français langue première ("à la française"). 

tuteurs. Comme le souligne Cicurel, "l'action professorale est avant tout une action sur autrui, voulant produire des effets (de connaissance) sur lui" (2011 : 49). À l'image de ce qui se passe dans les cours de langue en présentiel, l'action du tuteur cherche à avoir un effet sur l'état de connaissance de l'apprenant. Yann exprime ici ses buts pédagogiques et justifie son choix de l'activité :

\title{
Carnet de bord Yann, semaine 6 :
}

\begin{abstract}
Je me suis rendu compte que je ne pouvais que faire de la compréhension globale et fine. Je ne peux pas faire de systématisation car je ne donne pas un cours sur un point précis. Cependant je voulais que Larry puisse acquérir des savoir-faire et des savoir-être. J'ai réfléchi à transcrire l'oral du document pour aider l'étudiant, et puis je me suis dit qu'il était suffisamment facile à comprendre.
\end{abstract}

Le carnet de bord révèle ainsi des motifs d'action (Cicurel, 2007a : 215) qui n'apparaissent pas clairement dans les interactions en ligne. Arrivé à la semaine 6, le tuteur témoigne de l'évolution dans sa réflexion: "Je me suis rendu compte". Cet extrait manifeste l'écart entre ce que Yann aimerait faire, et qu'il estime possible en présentiel, et ce qu'il peut faire à distance. En ce sens, le tutorat en ligne apparait comme un obstacle. Toutefois, le tuteur exprime clairement sa volonté et envisage différentes solutions possibles pour parvenir à son but.

\section{Une adaptation aux goûts et aux demandes de l'apprenant}

L'adaptation ne concerne pas uniquement le niveau de compétence que les tuteurs perçoivent. Yann et Sabrina revendiquent également une adaptation aux goûts exprimés par leur apprenant :

\section{Carnet de bord Yann, semaine 5 :}

A force de lui poser des questions et de recevoir des réponses, on a appris à connaître certains de ses goûts et de ses attentes pour son année en France l'an prochain, du coup nous construisons nos activités et témoignages suivant ses réponses et ses questions pour l'aider.

Avec la locution adverbiale "du coup", le tuteur manifeste clairement le lien qu'il perçoit entre les interactions avec l'apprenant, qui leur permettent de mieux le connaître, et l'élaboration d'activités spécialement conçues pour lui. Le motif d'action exprimé ici est toujours le même : aider leur apprenant. Les thèmes développés par les tuteurs sont ainsi directement liés aux demandes exprimées par Larry :

Carnet de bord Yann, semaine 6 :

Pour le dernier thème abordé, nous avons choisi les études car Larry vient étudier en France l'an prochain et il nous a demandé dans ses messages qu'on lui en parle.

\section{Carnet de bord Sabrina, semaine 5 :}

Je lui ai aussi envoyé un mail informel lui parlant un peu de Paris et de ses arrondissements, comme il nous l'avait demandé à Yann et moi.

Ces extraits témoignent de la forte présence de la causalité ("car", "comme") dans les textes des tuteurs qui exposent leurs motifs d'action. L'agir tutoral de Sabrina comme de 
Yann se caractérise par une très forte personnalisation des tâches, à l'image de cours particuliers.

\section{La collaboration entre les tuteurs}

31 L'autre spécificité du dispositif tient à la collaboration entre les tuteurs. Dans leur carnet de bord, Sabrina et Yann font état de leur répartition du travail, qui repose sur une alternance des rôles :

\section{Carnet de bord Sabrina, semaine 1 :}

Lorsque l'on travaille en groupe, il y a toujours un moment où l'on essaye de s'organiser pour répartir également le travail, et nous avons décidé d'alterner notre travail chaque semaine.

\section{Carnet de bord Yann, semaine 2 :}

Nous nous sommes mis d'accord avec Sabrina pour que l'un de nous écrive un témoignage toutes les deux semaines et l'autre trouve des articles de journaux toutes les deux semaines, cela permet de changer chaque semaine.

Les deux tuteurs verbalisent le moment d'organisation des tâches. Sabrina fait entrer ce phénomène dans des schèmes déjà connus ("il y a toujours") avant de l'ancrer spécifiquement dans l'interaction avec son binôme. Au-delà de l'organisation matérielle, Yann insiste sur l'étayage apporté par le travail collaboratif. Il demande l'avis de Sabrina pour choisir entre différents documents. Le choix des tâches se réalise donc de manière concertée :

\section{Carnet de bord Yann, semaine 2 :}

J'ai toujours peur que l'apprenant ne comprenne pas le sens du document ou que le vocabulaire soit trop compliqué ou encore que l'article soit trop long pour son niveau de lecture. C'est pourquoi j'en ai choisi plusieurs pour les montrer à Sabrina, après les questions de compréhensions et l'approche du thème des transports seront essentiels à sa compréhension.

Dans cet extrait, Yann exprime une émotion, celle de la peur, qu'il associe à l'adverbe " toujours". Cela signifie que le tuteur fait entrer ce dispositif dans ses expériences précédentes d'enseignement. Il manifeste trois risques ("que l'apprenant ne comprenne pas le sens du document ou que le vocabulaire soit trop compliqué ou encore que l'article soit trop long pour son niveau de lecture") et fait le lien entre cette appréhension et son interaction avec Sabrina. Les échanges au sein du binôme apparaissent ainsi pour lui comme une façon de se rassurer.

34 Cette discussion entre tuteurs est ancrée dans leurs pratiques, comme en témoigne cet extrait qui rapporte des routines :

Carnet de bord Yann, semaine 3 :

Sinon je pense que nous sommes maintenant bien organisés avec Sabrina pour préparer les activités et les témoignages à temps. C'est agréable de travailler comme ça, moins stressant: on discute et critique nos travaux respectifs pendant le cours avant de co-écrire le mail à Larry contenant les activités. 
35

semaine 3, Yann rend compte d'une régularité : "nous sommes maintenant bien organisés

". Cet extrait révèle l'évolution dans les émotions du tuteur: on passe de la peur à quelque chose d'agréable" et de "moins stressant", grâce à la collaboration au sein du binôme.

Nous avons cherché à montrer dans cette première partie de l'analyse que l'action des tuteurs se fabrique en coopération avec l'apprenant et l'autre tuteur. En ce sens, on peut considérer que les interactions jouent un grand rôle dans la fabrique de l'action tutorale à distance. Nous allons à présent nous demander sur quelles ressources cet agir tutoral se fonde.

\section{L'analogie, fondement de l'agir tutoral}

Cette partie consistera à étudier la façon dont les tuteurs construisent une analogie entre ce dispositif nouveau pour eux et leurs précédentes expériences présentant une forme d'analogie. L'importance du "passé de l'acteur" est mise en avant par Cicurel \& Rivière (2008 : 269). Tardif \& Lessard soulignent également l'influence chez le travailleur "de sa propre histoire de vie, de sa trajectoire à la fois personnelle et sociale, de sa formation antérieure, etc." (1999: 375-376). Nous avons montré à travers l'analyse d'entretiens d'autoconfrontation que l'expérience de l'enseignant influence largement son agir (Muller, 2013b). De la même manière, les apprentis tuteurs font entrer leur nouvelle expérience dans les schèmes qu'ils connaissent. Ils s'appuient sur leurs expériences passées pour construire leur action tutorale.

\section{Un discours sur l'action}

Comme les entretiens d'auto-confrontation, les carnets de bord se caractérisent par un dire sur le faire, ou un dire sur le dire : les tuteurs commentent leur pratique. Bien qu'ils n'aient pas été confrontés à des traces de leur action, il est possible d'observer une correspondance entre les énoncés des tuteurs adressés aux apprenants sur un forum de discussion et leurs commentaires a posteriori. L'action est traduite en discours (Cicurel, 2011: 254); des phases de description de l'action, de jugement et de catégorisation apparaissent. Les tuteurs construisent des significations, ils interprètent leurs comportements à la manière d'enseignants commentant la vidéo de leur cours (Cicurel, $2005: 21)$.

Il est intéressant de relever que les verbes "parler" et "dire", originellement utilisés pour renvoyer à des interactions orales, sont ici utilisés pour rendre compte d'actions écrites ${ }^{10}$

Carnet de bord Sabrina, semaine 1 :

Aujourd'hui, nous avons aussi envoyé notre réponse à Larry, lui disant que nous ne connaissions pas Hong Kong, et que nous avions hâte d'échanger avec lui.

\section{Carnet de bord Sabrina, semaine 6 :}

Je lui ai parlé de mon parcours, de mes choix, et c'était aussi l'occasion de lui parler du principe de mobilité des études en France.

\section{Carnet de bord Yann, semaine 2 :}


J'ai été le premier à écrire un témoignage, l'envoi sur internet s'est bien passé et la réponse de Larry (c'est comme ça qu'il préfère qu'on l'appelle) est vite arrivée avec un travail sérieux que nous allons corriger lors de la séance de cours.

Cet emploi manifeste la façon dont les tuteurs inscrivent cette nouvelle expérience dans le cadre d'expériences passées fondées sur des échanges didactiques à l'oral. Cependant, le verbe "écrire", qui rend bien compte d'interactions écrites asynchrones, est également employé. Pour se référer au suivi, Yann utilise le verbe "corriger". La transmission des tâches étant une action fondamentale dans le tutorat, on retrouve des occurrences du verbe "envoyer".

\section{La formation reçue}

41 Comme le soulignent Pastré, Mayen \& Vergnaud, "Les adultes se développent au cours de leur expérience professionnelle, et au cours des formations initiales et continues qu'ils reçoivent" $(2006: 148)$.

42 Dans ces extraits, les tuteurs se réfèrent explicitement à la formation qu'ils ont reçue :

\section{Carnet de bord Yann, semaine 2 :}

Pour la séance prochaine, c'était à mon tour de chercher des articles ou documents authentiques, j'essaye de me souvenir de documents similaires que j'ai pu étudier lors de mon apprentissage d'autres langues.

\section{Carnet de bord Sabrina, semaine 2 :}

Cela a vraiment été très motivant de recevoir le premier travail de Larry car on a envie de faire encore plus d'effort dans la réalisation de nos activités, de faire des choses plus élaborées, et de mettre en pratique tout ce qu'on a appris en cours pour construire les meilleurs supports d'activité possible.

Yann puise dans son expérience d'apprenant de langue lorsqu'il doit trouver des documents authentiques. L'analogie est mise en évidence par l'emploi de l'adjectif " similaire". Sabrina, quant à elle, ne se réfère pas à ses cours de langue mais à ses cours de didactique du FLE et souligne sa volonté et son enthousiasme de "mettre en pratique tout ce qu'on a appris en cours". Pour construire son agir tutoral dans ce dispositif inédit, elle s'appuie ainsi sur les enseignements qu'elle a suivis dans le cadre de son master. Il est intéressant de noter que ces deux remarques interviennent au début de la rédaction du carnet de bord (semaine 2). C'est donc dans un premier temps que les tuteurs se réfèrent explicitement à leur formation. C'est la ressource dans laquelle ils puisent le plus spontanément.

\section{Une expérience nouvelle, comparée à des expériences précédentes}

Sabrina, qui a eu l'occasion d'être tutrice en présentiel, compare cette expérience précédente au nouveau dispositif auquel elle est confrontée :

\section{Carnet de bord Sabrina, semaine 1 :}

Ce travail en binôme est aussi une nouveauté pour moi en ce qui concerne le tutorat ou l'enseignement car l'enseignement dispensé ne sera pas seulement le mien, la relation que j'aurais avec Larry ne sera pas réellement 
la mienne, mais il me connaitra seulement comme faisant parti du binôme avec Yann. C'est assez différent de ce que j'ai pu connaitre lors de mon expérience précédente de tutorat. le caractère collaboratif du tutorat, source de changement dans la relation didactique, qui semble singulier à Sabrina. Cet extrait, qui apparaît dès la première séance, témoigne des points de référence sur lesquels la tutrice s'appuie. C'est par comparaison avec ses expériences précédentes qu'elle caractérise le tutorat en ligne. En ce sens, on peut considérer que la pratique antérieure des tuteurs constitue une ressource à partir de laquelle ils construisent un agir tutoral à distance.

\section{Reprise de routines antérieures}

C'est ainsi que Sabrina se fonde sur des routines ${ }^{11}$ construites antérieurement pour agir dans ce dispositif précis :

Carnet de bord Sabrina, semaine 7 :

47 À la fin de la télécollaboration, elle rend compte dans son carnet de bord de propos qu'elle a adressés à Larry. La tutrice évalue positivement cette démarche. Elle justifie cela plus loin en expliquant qu'elle a déjà eu recours à cette façon de procéder dans le cadre du tutorat en présentiel qu'elle a assuré : "C'est aussi ce que je fais avec mes anciens étudiants chinois". Son agir tutoral en présentiel prend ainsi largement appui sur ses expériences précédentes. Est-ce à dire qu'aucune nouvelle pratique ne peut apparaître?

\section{Élaboration de routines et émergence de nouvelles pratiques}

Dans cette dernière partie de l'analyse, nous allons chercher à montrer comment les tuteurs en ligne construisent des routines à partir des pratiques qu'ils mettent en œuvre.

\section{D'un tâtonnement à la construction de routines}

49 À la deuxième semaine, Sabrina emploie le terme "tâtonnement" pour caractériser les premières séances :

Carnet de bord Sabrina, semaine 2 :

Les premières séances de tutorat sont en quelque sorte un tâtonnement afin de mieux estimer le niveau de Larry.

Mais on peut remarquer à la lecture des carnets de bord que des routines s'installent rapidement. Nous entendons ici par routines des "structures d'action récursives et stables, à la fois liées à la maîtrise des situations de travail en classe et à la construction de l'identité 
professionnelle de l'enseignant" (Tardif \& Lessard, 1999: 387). C'est donc à travers l'expérience que peuvent se construire des routines. Pour Tochon, il s'agit de "plans intériorisés par l'expérience, automatisés" (1993 : 93). Ces routines constituent des ressources pour l'agir.

51 Dès la semaine 3, Yann considère qu'il est entré dans un processus régulier :

Carnet de bord Yann, semaine 3 :

Les échanges sont maintenant bien lancés, nous communiquons régulièrement. Je regarde le site des carnets quelques fois dans la semaine.

Il a recours au présent d'habitude pour rendre compte du fonctionnement du tutorat : " nous communiquons", "Je regarde". La rapidité avec laquelle les enseignants peuvent dire " c'est ainsi que nous avons toujours fait"12 est également soulignée par Woods (1996 : 266).

53 À la semaine 5, Sabrina fait également part de structures stables établies :

\section{Carnet de bord Sabrina, semaine 5 :}

Je trouve aussi que l'on s'est bien réparti le travail, car les activités que nous faisons pour Larry sont très fluides, préparées, mais ne nous débordent pas car c'est aussi une bonne gestion de groupe que l'on a réussi à trouver.

Ce sont des schèmes que les tuteurs ont intériorisés tout au long du dispositif et qui se sont construits progressivement: "c'est aussi une bonne gestion de groupe que l'on a réussi à trouver". Yann rend lui aussi compte de routines dans le travail à deux. Le recours à l'adjectif "même" à trois reprises et au déterminant "chaque" manifeste la régularité qui a caractérisé selon lui le tutorat:

Carnet de bord Yann, semaine 7 :

Le travail en binôme avec Sabrina s'est très bien passé, nous avons gardé la même manière de travailler tout le long, alternant les rôles chaque semaine, gardant le même système de correction et le même rythme d'échanges.

Vers la fin des carnets de bord, aux semaines 6 et 7, apparaissent des références à l'habitude :

\section{Carnet de bord Yann, semaine 6 :}

Du coup, la manière de préparer les activités était différente que d'habitude pour les documents écrits comme les articles de journaux ou sites internet.

\section{Yann, semaine 7 :}

Je profitais d'un accès internet sur mon lieu de travail le samedi et le lundi pour consulter rapidement le site des Carnets et voir si Larry avait des problèmes ou s'il avait déjà eu le temps de faire les activités. Je préparais les activités le lundi ou mardi soir en cherchant des articles sur internet ou en écrivant un témoignage.

Yann met en évidence les différences entre la tâche qu'il propose à la semaine 7 et celles conçues les semaines précédentes. L'emploi de la locution "d'habitude" révèle le fonctionnement ordinaire que les tuteurs ont intériorisé. La semaine suivante, Yann a recours à l'imparfait d'habitude pour rendre compte de son agir tutoral : "Je profitais", "Je préparais". Des routines sont ainsi élaborées même en ce qui concerne les jours consacrés à la préparation. 
L'usage des outils numériques a également conduit à l'instauration de routines chez les tuteurs :

\section{Carnet de bord Sabrina, semaine 6 :}

Malgré les quelques problèmes techniques que l'on a pu rencontrer avec Yann, l'usage des carnets m'est de plus en plus facile. Les procédures sont très simples et à force de faire la même chose chaque semaine, je commence à aller plus automatiquement vers les rubriques habituelles et même quelques fois vers d'autres que je ne connais pas.

58 À la semaine 6, Sabrina fait part de la facilité grandissante avec laquelle elle manie l'utilisation des Carnets 2 Paris Descartes. C'est grâce aux structures régulières répétées qu'elle a pu s'approprier l'outil : "à force de faire la même chose chaque semaine". L'usage du déterminant "chaque" est ici encore significatif. On observe ainsi la façon dont ce dispositif nouveau fait l'objet d'une appropriation par les tuteurs qui construisent très vite des routines, bien que le projet n'ait duré que 8 semaines.

\section{Émergence de nouvelles pratiques}

59 Si les tuteurs font état de routines dans le processus du tutorat, ils mettent également en avant l'émergence de nouvelles pratiques :

\section{Carnet de bord Yann, semaine 4 :}

Pour la semaine prochaine, j'ai essayé de lui faire faire quelque chose de nouveau et risqué : Poster une critique de film sur le site allociné.fr. J'espère qu'il n'aura pas de problème technique pour effectuer cette tâche. Mais je pense que c'est intéressant, surtout s'il vient étudier en France l'an prochain; il connaitra une référence en matière d'information sur le cinéma en France (c'est un thème qu'il aime particulièrement) et ça lui apprendra à utiliser un site internet en France qui sera indispensable pendant son séjour.

Dès la semaine 4, Yann écrit : "j'ai essayé de lui faire faire quelque chose de nouveau et risqué". L'usage de l'adjectif "nouveau" signifie qu'il a déjà intégré des routines et qu'il perçoit les écarts entre ces routines nouvellement construites et d'autres pratiques. L'approche nouvelle en question consiste à demander à Larry de publier une critique de film sur le site AlloCiné. Le tuteur novice justifie et argumente l'intérêt de cette tâche: "c'est intéressant", "ça lui apprendra". Il convient de s'interroger sur la notion de risque exprimée. Comme le souligne Cicurel (2007b), l'enseignement, même s'il comporte une part de routine, peut être considéré comme une action "risquée" en raison notamment de la possibilité de perdre la face. C'est peut-être ce qu'exprime ici Yann : le risque sera que Larry ne réalise pas la tâche et que le concepteur et tuteur se trouve remis en cause.

61 À la semaine 6, l'expression "quelque chose de nouveau" apparaît à nouveau :

\section{Carnet de bord Yann, semaine 6 :}

Et pour les dernières activités, $\mathbf{j}$ 'ai fait quelque chose de nouveau : de la compréhension orale à partie d'un document audio / vidéo. J'ai d'abord cherché des documents authentiques sur TV5 ou RFI puis je suis allé sur le site de l'INA qui est ma foi très bien pour la pédagogie. J'ai choisi un reportage télévisé sur un candidat au BAC âgé de 81 ans. La narration est facile à comprendre et le ton légèrement humoristique. Au début j'ai pensé choisir une vidéo d'époque (par exemple sur la mixité à l'école à la fin des 
années 60) puis j'ai préféré choisir un document récent pour que Larry ait une image de la France d'aujourd'hui. explicite sa démarche et se positionne sur les ressources ("qui est ma foi très bien pour la
pédagogie"), ce qui témoigne de l'expérience qu'il a acquise dans l'évaluation des
documents à exploiter. Il justifie son choix de la vidéo en exprimant un motif d'action: "
pour que Larry ait une image de la France d'aujourd'hui".

\section{Conclusion}

Lanalyse du corpus révèle la façon dont ces discours réflexifs des tuteurs témoignent "en direct" de la fabrique de l'action enseignante. Certes, il y a bien nécessairement un écart entre le discours sur les pratiques et les pratiques effectives, mais il semblerait que l'on assiste à la construction d'un répertoire didactique à distance. Les tuteurs se saisissent de ces écrits réflexifs pour réfléchir à leur expérience. Trois éléments apparaissent fondamentaux dans cet agir tutoral à distance. Il s'agit tout d'abord des interactions avec l'apprenant et l'autre tuteur. Que ce soit à distance avec l'étudiant hongkongais ou en présentiel avec le binôme, les échanges et la collaboration font partie intégrante de l'agir des tuteurs. Les similitudes que les participants perçoivent avec leurs expériences antérieures jouent ensuite un grand rôle, qu'il s'agisse de pratiques enseignantes, tutorales ou apprenantes. L'analogie pourrait ainsi être considérée comme un moteur fondamental dans la construction de l'action enseignante. Le troisième élément concerne l'émergence de nouvelles pratiques qui donnent lieu à l'installation de routines. Ce phénomène, relativement inattendu en raison de la courte durée du projet, témoigne de la façon dont les apprentis tuteurs ont intégré les modèles implicites qu'ils ont partiellement conçus.

Dans le cadre de cette étude exploratoire, fondée sur l'analyse de deux carnets de bord, nous avons pu apporter quelques éléments concernant les représentations de l'enseignant-tuteur en ligne face à sa nouvelle tâche, mais également concernant le développement professionnel de futurs enseignants de FLE. Il convient bien entendu de prendre en compte les spécificités du dispositif, à savoir les proportions exceptionnelles de deux tuteurs pour un apprenant, ainsi que le fait que les tuteurs soient novices. Il serait donc intéressant d'étendre l'étude à d'autres contextes afin de caractériser l'action enseignante à distance, de décrire des pratiques de transmission propres à l'enseignement ou au tutorat à distance et de faire émerger des styles d'enseignement et de tutorat.

Nous avons développé le point de vue des tuteurs, mais la fabrique de l'action apprenante mériterait également d'être explorée, notamment la fabrique de l'action apprenante à distance. Il s'agirait de recueillir les verbalisations d'apprenants sur de tels dispositifs impliquant les outils numériques, à travers des entretiens ou des écrits réflexifs. Cela permettrait de développer davantage le positionnement des apprenants et leur perception du dispositif. Les participants pourraient commenter, décrire et interpréter leur action. L'analyse de la mise en mots de leur expérience viserait à acquérir une meilleure compréhension des dispositifs à distance en intégrant le point de vue émique des apprenants.

Recherches en didactique des langues et des cultures, 12-2 | 2015 


\section{BIBLIOGRAPHIE}

Bailey, K. (1990). "The use of diary studies in teacher education programs". In Richards, J. C. \& Nunan, D. (dir.). Second Language Teacher Education. Cambridge : Cambridge University Press, pp. 215-226.

Bertin, J.-C. (2013). "Distance et acteurs de la situation pédagogique : une approche par l'ergonomie didactique - le cas de l'apprentissage des langues". In Annoot, E. \& Bertin, J.-C. (dir.). L'Homme @ Distance : Innovation et Développement - Regards croisés. Paris : CNRS éditions, pp. 113-137.

Borg, S. (2003). "Teacher cognition in language teaching: A review of research on what language teachers think, know, believe, and do". Language teaching, n 36(2), pp. 81-109.

Borg, S. (2009). "Introducing language teacher cognition". http://www.education.leeds.ac.uk/ research/files/145.pdf.

Bourdet, J.-F. (2006). "Construction d'un espace virtuel et rôles du tuteur". Le français dans le monde-Recherches et applications, pp. 32-43.

Cambra Giné, M. (2003). Une approche ethnographique de la classe de langue. Paris : Didier.

Cicurel, F. (2005). "La flexibilité communicative. Un atout pour la construction de l'agir enseignant". Le français dans le monde - Recherches et applications, juillet 2005, pp. 180- 191.

Cicurel, F. (2007a). "À la recherche d'une grammaire de l'agir professoral". In Charolles, M., Fournier, N., Fuchs, C. \& Lefeuvre, F. (dir.). Parcours de la phrase. Mélanges offerts à Pierre le Goffic. Paris : Ophrys, pp. 213-225.

Cicurel, F. (2007b). "L'agir professoral, une routine ou une action à haut risque ?". In Plazaola Giger, I \& Stroumza, K. (dir.). Paroles de praticiens et description de l'activité. Problématisation méthodologique pour la formation et la recherche. Bruxelles : De Boeck, pp. 15-36.

Cicurel, F. (2011). Les interactions dans l'enseignement des langues - Agir professoral et pratiques de classe. Paris : Didier.

Cicurel, F. \& Rivière, V. (2008). "De l'interaction en classe à l'action revécue : le clair-obscur de l'action enseignante". In Filliettaz, L. \& Schubauer-Leoni, M.-L. (dir.). Processus interactionnels et situations éducatives. Bruxelles : De Boeck, pp. 255-273.

Dejean-Thircuir, C. \& Mangenot, F. (2006). "Les échanges en ligne dans l'apprentissage et la formation : Cadrage et présentation". Le français dans le monde - Recherches et applications, pp. 5-13.

Denis, B. (2003). "Quels rôles et quelle formation pour les tuteurs intervenant dans des dispositifs de formation à distance ?". Distances et savoirs, vol. 1 n 1, pp. 19-46.

Develotte, C., Mangenot, F. \& Zourou, K. (2007). "Learning to Teach Online : 'Le français en (première) ligne' Project". In O’Dowd, R. (dir.). Online Intercultural Exchange. Clevedon, Buffalo, Toronto : Multilingual Matters, pp. 276-280.

Guichon, N. (2011). "Former les futurs enseignants de langue en ligne par le biais de la rétrospection". Alsic, vol. 14. URL : http://alsic.revues.org/1983.

Guichon, N. (2012). Vers l'intégration des TIC dans l'enseignement des langues. Paris : Didier. 
Mangenot, F. (2011). "Du e-learning aux interactions pédagogiques en ligne". In Nissen, E., Poyet, F. \& Soubrié, T. (dir.). Interagir et apprendre en ligne. Grenoble : Ellug, Université Stendhal, pp. 7-19.

Muller, C. (2013a). "'Tout ce que nous faisions me paraissait virtuel". Ou comment les conceptions de l'interaction verbale chez des tuteurs à distance influencent leur perception d'un dispositif de télécollaboration". Les Cahiers de l'Acedle, vol. 10, n², pp. 3-26. http://acedle.org/spip.php? article3722.

Muller, C. (2013b). "De l'influence de l'expérience sur l'agir enseignant". Éducation permanente, n - 196, pp. 129-138.

Muller, C. (2014). "Feedback et relation interpersonnelle apprenants/tuteurs dans un projet de

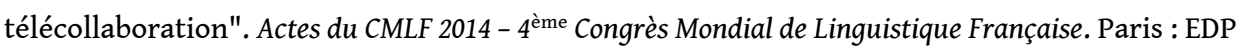
Sciences, Institut de Linguistique française.

Nissen, E. (2011). "Analyser les rôles du tuteur dans une formation hybride : distinguer les interactions verbales à distance et en présentiel". In Nissen, E., Poyet, F. \& Soubrié, T. (dir.). Interagir et apprendre en ligne. Grenoble : Ellug, Université Stendhal, pp. 147-166.

Pastré, P., Mayen, P. \& Vergnaud, G. (2006). "La didactique professionnelle". Revue française de pédagogie, $\mathrm{n}^{\circ} 154$, pp. 145-198.

Rivière, V. \& Guichon, N. (2014). "Construction de bilans rétroactifs par des apprentis tuteurs de langue en ligne : essai de caractérisation discursive de la dynamique socio-cognitive". Le français dans le monde - Recherches et applications, $\mathrm{n}^{\circ}$ 56, pp. 118-135.

Rodet, J. (2011). "Formes et modalités de l'aide apportée par le tuteur". In Depover, C., De Lièvre, B., Peraya, D., Quintin, J-J. \& Jaillet, A. (dir.). Le tutorat en formation à distance. Bruxelles : Editions De Boeck, pp. 159-170.

Sensevy, G. \& Mercier, A. (dir.) (2007). Agir ensemble. L'action conjointe du professeur et des élèves dans le système didactique. Rennes : PUR.

Tardif, M. \& Lessard, C. (1999). Le travail enseignant au quotidien. Expérience, interactions humaines et dilemmes professionnels. Bruxelles : De Boeck Université.

Tochon, F. V. (1993). L'enseignant expert. Paris : Nathan.

van Lier, L. ([1988] 1990). The Classroom and the Language Learner. Ethnography and Second-Language Classroom Research. Londres : Longman.

van Lier, L. (1996). Interaction in the Language Curriculum. Awareness, Autonomy and Authenticity. Londres : Longman.

Woods, D. (1996). Teacher Cognition in Language Teaching. Beliefs, decision-making and classroom practice. Cambridge : Applied Linguistics.

\section{Sites Internet}

AUF Appui à l'enseignement du français : http://www.aidenligne-francais-universite.auf.org/ spip.php?page=sommaire_appui_ens_fr

Le français en (première) ligne : http://fle-1-ligne.u-grenoble3.fr/

Les Carnets 2 Paris Descartes : http://carnets.parisdescartes.fr/ 


\section{NOTES}

1. Français langue étrangère.

2. "the teachers' perceptions and interpretations of the classroom events".

3. "an ethnographic study of teachers".

4. "understanding what teachers think, know and believe".

5. Ce partenariat a été créé grâce à l'aide de l'Agence universitaire de la francophonie, appui à l'enseignement du français, qui nous a mise en relation avec Céline Dias, enseignante de français à l'université baptiste de Hong Kong.

6. Pour une présentation plus détaillée du dispositif, voir par exemple Muller (2014).

7. Pour la perception des échanges par les tuteurs en lien avec leur approche des interactions verbales, le lecteur pourra se référer à Muller (2013a).

8. Tous les prénoms ont été modifiés.

9. Rivière \& Guichon (2014) soulignent l'intérêt d'étudier les discours d'apprentis tuteurs dont les normes sont encore en construction et dont le répertoire didactique n'est "pas encore stabilisé" $(2014: 132)$.

10. Nous avons ajouté des caractères en gras dans le corpus pour souligner les phénomènes saillants de l'analyse. Les extraits n'ont pas été corrigés.

11. L'importance des routines dans la pensée enseignante est notamment soulignée par Woods (1996: 178).

12. "we've always done it that way".

\section{RÉSUMÉS}

Dans cet article, nous nous intéressons à la façon dont des tuteurs en ligne, encore en formation, construisent leurs pratiques dans le cadre d'un projet de télécollaboration avec des apprenants de français langue étrangère. C'est le point de vue des tuteurs qui est ici privilégié. Le tutorat en ligne constituant une expérience inédite pour eux, on peut se demander comment ils élaborent un agir tutoral. Comment perçoivent-ils leur propre action ? Dans quelles ressources puisent-ils? Leur approche est-elle amenée à évoluer? Les tuteurs peuvent-ils faire appel à des routines antérieures? En construisent-ils de nouvelles? Comment de nouvelles pratiques peuvent-elles émerger? Pour répondre à ces questions, nous analysons les écrits réflexifs (carnets de bord) d'un duo de tuteurs ayant peu d'expérience d'enseignement. Après avoir replacé nos interrogations dans la perspective des recherches sur l'agir enseignant, nous présentons le contexte et les modalités des échanges (interactions écrites asynchrones). Trois phénomènes font l'objet d'une analyse : la construction d'une action conjointe avec l'apprenant et avec le binôme, la reconnaissance d'analogies avec d'autres expériences, l'élaboration de routines à partir de l'émergence de nouvelles pratiques.

This article analyses the way in which online tutors, who are still student teachers, construct their practices in the context of a telecollaboration project with French language learners. I focus here on the tutors' points of view. Online tutoring being a totally new experience for them, how do they build a tutoring repertoire? How do they perceive their own action? What resources do 
they use? Are their approaches changing throughout the project? Can tutors use former routines? Do they develop new ones? How can new practices emerge? To answer these questions, I analyse reflective texts (logbooks) written by two tutors working together and having limited teaching experience. These issues are explored from the perspective of teacher cognition. I present the context and the nature of the exchanges (asynchronous written online interaction) before analysing three phenomena: the construction of joint action within each group made up of one learner and two tutors, the recognition of analogies with other experiences, the development of routines from the emergence of new practices.

\section{INDEX}

Mots-clés : télécollaboration, tutorat en ligne, pensée enseignante, routine

Keywords : telecollaboration, online tutoring, teacher cognition, routine

\section{AUTEUR}

\section{CATHERINE MULLER}

Université Grenoble Alpes, Lidilem, F-38040, Grenoble, France

Catherine Muller est maître de conférences en sciences du langage à l'Université Stendhal Grenoble 3 et membre de l'équipe Lidilem. Ses recherches portent sur le rapport à soi et le rapport aux autres dans l'enseignement/apprentissage des langues. Cela la conduit à aborder les dimensions socio-affectives, la relation didactique, l'agir professoral et l'interculturalité en analysant des interactions didactiques en présentiel ou à distance, ainsi que des discours réflexifs d'enseignants, novices ou chevronnés, et d'apprenants de langues.

Toile: https://sites.google.com/site/catherinemuller9/

Courriel: catherine.muller9[at]gmail.com 